\title{
ON PRODUCT-FORM STATIONARY DISTRIBUTIONS FOR REFLECTED DIFFUSIONS WITH JUMPS IN THE POSITIVE ORTHANT
}

\author{
FRANCISCO J. PIERA, * Purdue University \\ RAVI R. MAZUMDAR, ${ }^{* *}$ University of Waterloo and Purdue University \\ FABRICE M. GUILLEMIN, ${ }^{* * *}$ France Télécom
}

\begin{abstract}
In this paper, we study the stationary distributions for reflected diffusions with jumps in the positive orthant. Under the assumption that the stationary distribution possesses a density in $\mathbb{R}_{+}^{n}$ that satisfies certain finiteness conditions, we characterize the FokkerPlanck equation. We then provide necessary and sufficient conditions for the existence of a product-form distribution for diffusions with oblique boundary reflections and jumps. To do so, we exploit a recent characterization of the boundary properties of such reflected processes. In particular, we show that the conditions generalize those for semimartingale reflecting Brownian motions and reflected Lévy processes. We provide explicit results for some models of interest.
\end{abstract}

Keywords: Diffusion; jump; reflection map; local time; semimartingale; stationary distribution; product form

2000 Mathematics Subject Classification: Primary 60J50; 60J55

Secondary 60J60; 60J75; 90B22

\section{Introduction}

Reflected diffusions with jumps arise in a wide variety of applications, such as finance, queueing and risk theory, and models of manufacturing plants. For example, in [7] the authors have shown that, in heavy traffic, the process corresponding to the number of customers in queues subject to service interruptions can be approximated by a reflected Brownian motion (RBM) with jumps. This was generalized to open queueing networks in [15], where convergence to a multidimensional reflected Lévy process was shown. More recently, such processes have also been shown to arise in diffusion limits involving jumps with heavy-tailed distributions [16]. In addition, reflected diffusion models with jumps are natural generalizations of the class of so-called piecewise-deterministic Markov processes [3], the generalization being that the diffusive component adds to the randomness of the evolution of the process between jumps, and reflections guarantee that the components of the process stay within a given region (for example in queueing networks where the processes are nonnegative). These models are also of interest in the risk and insurance context, where the jumps could be the claims while the

Received 28 April 2004; revision received 13 October 2004.

* Postal address: School of Electrical and Computer Engineering, Purdue University, West Lafayette, IN 47907-1285, USA. Email address: fpieraug@purdue.edu

** Postal address: Department of Electrical and Computer Engineering, University of Waterloo, Waterloo, Ontario N2L 3G1, Canada. Email address: mazum@ece.uwaterloo.ca

*** Postal address: France Télécom R\&D, 2 Avenue Pierre Marzin, 22300 Lannion, France.

Email address: fabrice.guillemin@francetelecom.com 
diffusion arises due to volatility of the interest rates, etc. They also play an important role in mathematical finance; in the context of barrier options, for example.

A special case of reflected diffusions, namely semimartingale reflecting Brownian motion (SRBM), has been studied quite extensively due to its importance in models of queueing networks in heavy traffic [4], [17], [18]. In [12], necessary conditions for the existence of SRBM in the positive orthant were given, in terms of a special condition on the reflection matrix called the completely-S property. In [14], the sufficiency and uniqueness under this condition were established. Moreover, in [12] it was also shown to be a boundary property, in that the reflection map does not charge the set of times spent by SRBM in the intersection of two or more faces. More recently, in [13] the authors used this property to develop numerical methods for computing the stationary distribution of queueing networks in the heavy traffic limit.

In [9], one-dimensional reflected diffusions with jumps, and their corresponding stationary distributions (when they exist), were studied. It was shown that not only is the Lebesgue measure of the set of times that the process spends at the origin equal to zero, but also that there is no probability mass at that point, Lebesgue almost everywhere in time. Recently, in [10], a characterization of the boundary properties of multidimensional reflected diffusions with jumps was obtained, generalizing those in [9]. The boundary characterization is essential in studying the properties of the stationary distribution of the process, when it exists.

There has been much interest in the particular case of when the marginal distributions are independent - so-called product-form distributions. Product-form distributions for stationary, reflected Lévy processes (i.e. with constant drift and diffusion matrix) have been exploited in [1], for example; however, no conditions guaranteeing this separability property were given. In [4], the authors provided necessary and sufficient conditions for the separability of the distribution in the stationary regime of SRBM, where there are no jumps and the drift, as well as the diffusion matrix, are constant. Negative results for queueing models with general nondecreasing, and possibly dependent, Lévy inputs were given in [6]. More recently, negative results in the context of Lévy stochastic networks were given in [8].

In this paper, we exploit the boundary characterization in [10] to study the stationary distributions of reflected diffusions with jumps in $\mathbb{R}_{+}^{n}$. In particular, we derive the forward, or Fokker-Planck, equation. We then study the conditions for the existence of product-form stationary distributions, assuming that the stationary setting exists, obtaining the necessary and sufficient conditions in the case of nonconstant drift and diffusion coefficients, and so generalizing the conditions found in [4] for the simpler case of SRBM, and in [6] and [8] for reflected Lévy processes.

The organization of the paper is as follows. In Section 2, we introduce the model and obtain preliminary results. In Section 3, we specialize these to the case where the stationary density is separable. In Section 4, we obtain our main result, regarding necessary and sufficient conditions for product-form distributions of reflected diffusions with jumps in the positive orthant in the stationary regime, assuming that the stationary setting and its corresponding density exist. In Section 5, we consider some examples of interest. Finally, Section 6 offers some further comments on the scope of our results.

\section{Problem formulation and preliminary results}

Let $n>1$ be a positive integer, let $\left(\Omega, \mathcal{F},\left(\mathscr{F}_{t}\right)_{t \geq 0}, \mathrm{P}\right)$ be a filtered probability space satisfying the usual hypotheses (see, e.g. [11]), and let $\mathbb{R}_{+}^{n}=\left\{\left(x_{1}, \ldots, x_{n}\right) \in \mathbb{R}^{n}: x_{i} \geq 0\right.$ for all $i=1, \ldots, n\}$ (a corresponding definition applies for $\mathbb{R}_{+}$, i.e. it includes 0 ). Note that, 
even though when explicitly writing elements in $\mathbb{R}^{n}$ or $\mathbb{R}_{+}^{n}$ we write them as row vectors (for simplicity of notation), they are treated as column vectors in all the equations in which they appear.

We consider a problem of reflection in $\mathbb{R}_{+}^{n}$ :

$$
\mathrm{d} X_{t}=b\left(X_{t-}\right) \mathrm{d} t+\sigma\left(X_{t-}\right) \mathrm{d} W_{t}+\int_{\mathbb{R}_{+}^{n}} z \Pi(\mathrm{d} t, \mathrm{~d} z)+R \mathrm{~d} Z_{t} .
$$

Our notation is as follows.

1. $\left(X_{t}\right)=\left(X_{t}^{1}, \ldots, X_{t}^{n}\right)$ is an $\left(\mathcal{F}_{t}\right)$-adapted, càdlàg, $\mathbb{R}_{+}^{n}$-valued semimartingale and $\left(X_{t-}\right)=$ $\left(\lim _{s \uparrow t} X_{s}\right)$, with $X_{0-}=X_{0}$ by convention.

2. $\left(W_{t}\right)=\left(W_{t}^{1}, \ldots, W_{t}^{n}\right)$ is an $n$-dimensional, $\left(\mathscr{F}_{t}\right)$-adapted, standard Brownian motion.

3. $\left(Z_{t}\right)=\left(Z_{t}^{1}, \ldots, Z_{t}^{n}\right)$ is an $\left(\mathscr{F}_{t}\right)$-adapted, continuous, $\mathbb{R}_{+}^{n}$-valued process such that for all $i \in\{1, \ldots, n\},\left(Z_{t}^{i}\right)$ is nondecreasing and null at zero, and $\int_{\mathbb{R}_{+}} X_{s}^{i} \mathrm{~d} Z_{s}^{i}=0$.

4. $b=\left(b_{i}\right)_{i \in\{1, \ldots, n\}}$ and $\sigma=\left(\sigma_{i, j}\right)_{i, j \in\{1, \ldots, n\}}$ are Borel-measurable mappings from $\mathbb{R}_{+}^{n}$ into $\mathbb{R}^{n}$ and $\mathbb{R}^{n^{2}}$, respectively. We set $a:=\sigma \sigma^{\top}$, where $\sigma^{\top}$ corresponds to the transpose of matrix $\sigma$. Furthermore, denoting by $a_{i, i}\left(0_{i}\right)$ the $i$ th diagonal element of matrix $a$ when its $i$ th coordinate has been set to 0 , we assume that for all $i \in\{1, \ldots, n\}, a_{i, i}\left(0_{i}\right)>0$ everywhere in $\mathbb{R}_{+}^{n-1}$.

5. $\Pi(\mathrm{d} t, \mathrm{~d} z)$ is an $\left(\mathcal{F}_{t}\right)$-adapted, $\{0,1\}$-valued random measure over $\mathbb{R}_{+} \times \mathbb{R}_{+}^{n}$. Furthermore, we assume that $\Pi(\mathrm{d} t, \mathrm{~d} z)$ admits a predictable compensator $\Pi^{\mathrm{p}}(\mathrm{d} t, \mathrm{~d} z)$ of the form $\lambda(\omega) \bar{K}(\omega, t, \mathrm{~d} z) \mathrm{d} t$, such that

(i) $\bar{K}(\omega, t, \mathrm{~d} z)$ is a Markovian, predictable transition kernel of $\left(\Omega \times \mathbb{R}_{+}, \mathcal{P}\right)$ into $\left(\mathbb{R}_{+}^{n}\right.$, $\left.\mathscr{B}\left(\mathbb{R}_{+}^{n}\right)\right)$ taking the form $K\left(X_{t-}(\omega), \mathrm{d} z\right)$, where $\mathcal{P}$ denotes the corresponding predictable $\sigma$-field on $\Omega \times \mathbb{R}_{+}, \mathcal{B}\left(\mathbb{R}_{+}^{n}\right)$ the collection of Borel sets in $\mathbb{R}_{+}^{n}$, and $K(x, \mathrm{~d} z)$ a transition kernel of $\left(\mathbb{R}_{+}^{n}, \mathcal{B}\left(\mathbb{R}_{+}^{n}\right)\right)$ into itself such that for all $x \in \mathbb{R}_{+}^{n}$, it admits (with respect to the Lebesgue measure) a density $k(x, z)$;

(ii) in the stationary setting (assuming that it exists), the intensity $\lambda(\omega)$ is jointly distributed with $X_{t-}(\omega)$ as $\psi(\mathrm{d} r, \mathrm{~d} x)$, where the variables $r \in \mathbb{R}_{+}$and $x \in \mathbb{R}_{+}^{n}$ refer to $\lambda$ and $X_{t-}$, respectively, and the joint law $\psi(\mathrm{d} r, \mathrm{~d} x)$ admits (with respect to the Lebesgue measure) a density $\varphi(r, x)$. Moreover, for all $x \in \mathbb{R}_{+}^{n}$, we write $\Phi(x):=\int_{\mathbb{R}_{+}} r \varphi(r, x) \mathrm{d} r$.

6. $R$ is an $n \times n$ P-matrix. Recall that a square matrix with real coefficients is said to be a $\mathrm{P}$-matrix if every principal minor is strictly positive. (Note that this condition is satisfied, for example, by any real triangular matrix $R$ with strictly positive diagonal elements and, more generally, by positive-definite matrices.) Hence, in particular, $R$ has strictly positive diagonal elements and is invertible. Also, note that P-matrices are completely-S (in the terminology of [12]) and, in addition, that their principal submatrices are invertible. (In a discrete-time setting, this invertibility condition guarantees that the linear complementarity problem associated with defining the reflection map has a unique solution (see, e.g. [2]).)

In addition, we assume hereafter that $b, \sigma, \Pi$, and $R$ are such that (1) has a unique strong solution. In particular, $\sigma$ and $b$ satisfy the usual local Lipschitz and linear growth conditions [5]. 
The following notation will be used throughout the paper. Let $\mathbb{N}$ be the set of strictly positive integers. Then, for $k \in \mathbb{N}, f: \mathbb{R}_{+}^{k} \rightarrow \mathbb{R}$, and $i \in\{1, \ldots, k\}$, we write $f\left(0_{i}\right)$ to indicate that the $i$ th coordinate in $f$ has been set to $0, f\left(0_{i}^{+}\right)$to denote $\lim _{x_{i} \downarrow 0} f(x)$, and $\mathcal{L}_{k}[f](\cdot)$ to denote the $k$-dimensional Laplace transform of function $f$. Furthermore, whenever we write 'almost everywhere in $\mathbb{R}_{+}^{k}$ ' or 'for almost every $x \in \mathbb{R}_{+}^{k}$ ', without specifying the measure, we mean it with respect to the Lebesgue measure in $\mathbb{R}_{+}^{k}$. Of course, when $k=1$, the indices $i$ and $k$ in all the previous notation are not necessary, and will be omitted. Moreover, for $i, j \in\{1, \ldots, n\},\left(\left[X^{i}, X^{j}\right]_{t}^{c}\right)$ denotes the path-by-path continuous part of the quadratic covariation process $\left(\left[X^{i}, X^{j}\right]_{t}\right)$, or of the quadratic variation process if $i=j$, and $\left(L_{t}^{i}\right)$ denotes the local time at level 0 for the semimartingale $\left(X_{t}^{i}\right)$. In addition, for $l, k \in \mathbb{N}$, we write $\mathcal{C}^{l}\left(\mathbb{R}_{+}^{k}\right)$ or $\mathcal{C}_{b}^{l}\left(\mathbb{R}_{+}^{k}\right)$ to indicate the set of all functions $f: \mathbb{R}_{+}^{k} \rightarrow \mathbb{R}$ that, along with all their partial derivatives up to and including order $l$, are continuous or, respectively, bounded and continuous in $\mathbb{R}_{+}^{k}$. Of course, when no superscript $l$ is specified in this last notation, we refer to $f$ itself, excluding its derivatives. Also, to simplify the expressions, for $k \in\{1, \ldots, n\}$ we write $\mathrm{d} x \neq k$ to indicate $\mathrm{d} x_{1} \cdots \mathrm{d} x_{n}$ with the $k$ th differential omitted, as well as $x_{\neq k}$ or $x+z_{k}$ to indicate that the $k$ th coordinate in $x \in \mathbb{R}_{+}^{n}$ is omitted or incremented by $z \in \mathbb{R}_{+}$, respectively. Furthermore, we write $\Gamma^{n}$ to denote the boundary faces of $\mathbb{R}_{+}^{n}$, i.e. $\bigcup_{i=1}^{n}\left\{x \in \mathbb{R}_{+}^{n}: x_{i}=0\right\}$. Finally, since throughout the paper we will just need to consider the index set $\{1, \ldots, n\}$, from now on we will omit it, writing, for example, 'for all $i$ ' instead of 'for all $i \in\{1, \ldots, n\}$ '.

Henceforth, we will assume that the stationary regime exists and that, in this regime, the law of $X_{t}$ in $\mathbb{R}_{+}^{n}$ admits a density (with respect to the Lebesgue measure), denoted by $p(x), x \in \mathbb{R}_{+}^{n}$. Note that, since for all $i, a_{i, i}\left(0_{i}\right)>0$ everywhere in $\mathbb{R}_{+}^{n-1}$, by [10, Lemma 2.1] applied to the stationary version of semimartingale $\left(X_{t}\right)$, there is no probability mass in the boundary faces of $\mathbb{R}_{+}^{n}$ (i.e. there is no probability mass in $\Gamma^{n}$ ) and, therefore, it is enough to consider a density with respect to the Lebesgue measure (in $\mathbb{R}_{+}^{n}$ ). Furthermore, we will assume that the following four conditions are satisfied for all $i$ and $j$.

(c1) $\int_{\mathbb{R}_{+}^{2 n}} z_{i} \Phi(x) k(x, z) \mathrm{d} z \mathrm{~d} x=\int_{\mathbb{R}_{+}^{n+1}} z_{i} \Phi(x) k_{i}\left(x, z_{i}\right) \mathrm{d} z_{i} \mathrm{~d} x<\infty$, where $k_{i}\left(x, z_{i}\right)$ denotes the $i$ th one-dimensional marginal density extracted from $k(x, z)$, for all $x \in \mathbb{R}_{+}^{n}$.

(c2) $\int_{\mathbb{R}_{+}^{n}}\left|b_{i}(x)\right| p(x) \mathrm{d} x<\infty$.

(c3) $\int_{\mathbb{R}_{+}^{n}}\left|a_{i, j}(x)\right| p(x) \mathrm{d} x<\infty$.

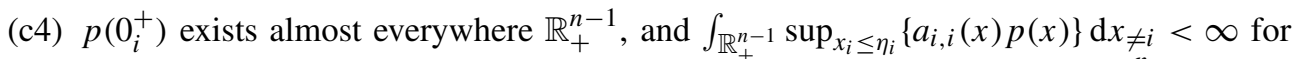
some $\eta_{i}>0$. Note that if $p$ is separable (i.e. if for almost every $x \in \mathbb{R}_{+}^{n}, p(x)=$ $\prod_{k=1}^{n} p_{k}\left(x_{k}\right)$, where, for all $k, p_{k}$ is the $k$ th one-dimensional marginal density extracted from $p$ ) and if, furthermore, $a_{i, i}(x)=a_{i, i}\left(x_{i}\right)$ for all $x \in \mathbb{R}_{+}^{n}$, then this condition reduces to the existence and finiteness of $p_{i}\left(0^{+}\right)$.

Condition (c1) guarantees that [11, p. 173, Hypothesis A] is satisfied in the stationary regime; conditions (c2) and (c3) allow the use of Fubini's theorem in subsequent proofs in the paper, as well as guaranteeing that the Laplace transforms of $b_{i} p$ and $a_{i, j} p$ exist for all $i, j$; and, finally, condition (c4) allows the use of some previously stated results of [10].

We define the operator $\mathcal{T}: \mathcal{C}^{2}\left(\mathbb{R}_{+}^{n}\right) \rightarrow \mathcal{C}\left(\mathbb{R}_{+}^{n}\right)$ as follows:

$$
\mathcal{T} f(x):=\sum_{i=1}^{n} b_{i}(x) \frac{\partial f}{\partial x_{i}}(x)+\frac{1}{2} \sum_{i, j=1}^{n} a_{i, j}(x) \frac{\partial^{2} f}{\partial x_{i} \partial x_{j}}(x) .
$$


The following lemmas will be used in subsequent sections of the paper.

Lemma 1. The following relationship holds for all $f \in \mathcal{C}_{b}^{2}\left(\mathbb{R}_{+}^{n}\right)$ :

$$
\begin{aligned}
0= & \int_{\mathbb{R}_{+}^{n}} \mathcal{T} f(x) p(x) \mathrm{d} x+\frac{1}{2} \sum_{i, j=1}^{n} \frac{R_{i, j}}{R_{j, j}} \int_{\mathbb{R}_{+}^{n-1}} \frac{\partial f}{\partial x_{i}}\left(0_{j}\right) a_{j, j}\left(0_{j}\right) p\left(0_{j}^{+}\right) \mathrm{d} x_{\neq j} \\
& +\int_{\mathbb{R}_{+}^{2 n}}\{f(x+z)-f(x)\} \Phi(x) k(x, z) \mathrm{d} z \mathrm{~d} x .
\end{aligned}
$$

Proof. Let $f \in \mathcal{C}_{b}^{2}\left(\mathbb{R}_{+}^{n}\right)$. Using Itô's formula [11, p. 74, Theorem 33], (1), and the fact that $\mathrm{d}\left[X^{i}, X^{j}\right]_{s}^{c}=a_{i, j}\left(X_{s-}\right) \mathrm{d} s$, we find that

$$
\begin{aligned}
f\left(X_{t}\right)-f\left(X_{0}\right)= & \sum_{i=1}^{n} \int_{0+}^{t} \frac{\partial f}{\partial x_{i}}\left(X_{s-}\right) b_{i}\left(X_{s-}\right) \mathrm{d} s+\sum_{i, j=1}^{n} \int_{0+}^{t} \frac{\partial f}{\partial x_{i}}\left(X_{s-}\right) \sigma_{i, j}\left(X_{s-}\right) \mathrm{d} W_{s}^{j} \\
& +\frac{1}{2} \sum_{i, j=1}^{n} \int_{0+}^{t} \frac{\partial^{2} f}{\partial x_{i} \partial x_{j}}\left(X_{s-}\right) a_{i, j}\left(X_{s-}\right) \mathrm{d} s+\sum_{0<s \leq t} \Delta f\left(X_{s}\right) \\
& +\sum_{i, j=1}^{n} \int_{0+}^{t} \frac{\partial f}{\partial x_{i}}\left(X_{s-}\right) R_{i, j} \mathrm{~d} Z_{s}^{j}
\end{aligned}
$$

where $\Delta f\left(X_{s}\right):=f\left(X_{s}\right)-f\left(X_{s-}\right)$. Assuming that we are working in the stationary regime, by taking expectations in the equation above we obtain

$$
0=t \int_{\mathbb{R}_{+}^{n}} \mathcal{T} f(x) p(x) \mathrm{d} x+\sum_{i, j=1}^{n} \mathrm{E} \int_{0+}^{t} \frac{\partial f}{\partial x_{i}}\left(X_{s-}\right) R_{i, j} \mathrm{~d} Z_{s}^{j}+\mathrm{E} \sum_{0<s \leq t} \Delta f\left(X_{s}\right),
$$

where, along with conditions (c2) and (c3) and Fubini's theorem, we have used the facts that $\left(X_{s}\right)$ is càdlàg $\left(\mathrm{P}\right.$ almost surely $(\mathrm{P}$-a.s. $)$ ), that the Lebesgue measure is diffuse, that $f \in \mathcal{C}_{b}^{2}\left(\mathbb{R}_{+}^{n}\right)$, and that the stochastic integrals involved are centred, continuous local martingales. Note that the remaining expectations in (3) are to be taken in the stationary regime. Now, by condition (c4) and [10, Theorem 3.2 and Corollary 2.1], we have

$$
\begin{aligned}
\sum_{i, j=1}^{n} \mathrm{E} \int_{0+}^{t} \frac{\partial f}{\partial x_{i}}\left(X_{s-}\right) R_{i, j} \mathrm{~d} Z_{s}^{j} & =\sum_{i, j=1}^{n} \mathrm{E} \int_{0+}^{t} \frac{\partial f}{\partial x_{i}}\left(X_{s-}\right) \frac{R_{i, j}}{2 R_{j, j}} \mathrm{~d} L_{s}^{j} \\
& =\frac{t}{2} \sum_{i, j=1}^{n} \frac{R_{i, j}}{R_{j, j}} \int_{\mathbb{R}_{+}^{n-1}} \frac{\partial f}{\partial x_{i}}\left(0_{j}\right) a_{j, j}\left(0_{j}\right) p\left(0_{j}^{+}\right) \mathrm{d} x_{\neq j} .
\end{aligned}
$$

Note that $X_{S-}$ can be replaced by $X_{S}$ in the right-hand side of the first equality above since, for all $j$, the random measure induced by $\left(L_{S}^{j}\right)$ in $\mathbb{R}_{+}$is diffuse, and $\left(X_{S}\right)$ is càdlàg, $\mathrm{P}$-a.s. Finally, since

$$
\mathrm{E} \sum_{0<s \leq t} \Delta f\left(X_{S}\right)=t \int_{\mathbb{R}_{+}^{2 n}}\{f(x+z)-f(x)\} \Phi(x) k(x, z) \mathrm{d} z \mathrm{~d} x,
$$

(2) follows after dividing all the previous expressions by $t>0$. The lemma is now proved. 
From Lemma 1, we can easily obtain the Fokker-Planck equation in the stationary setting, as stated in the following corollary.

Corollary 1. Assume that $\Phi$ is continuous in $\mathbb{R}_{+}^{n}$, that for all $i$ and $j, b_{i} p \in \mathcal{C}^{1}\left(\mathbb{R}_{+}^{n}\right)$ and $a_{i, j} p \in \mathcal{C}^{2}\left(\mathbb{R}_{+}^{n}\right)$, and that the $n$-dimensional Laplace transform of $\partial\left(a_{i, i} p\right) / \partial x_{i}$ exists. Then, for all $x \in \mathbb{R}_{+}^{n}$, we have

$$
0=-\sum_{i=1}^{n} \frac{\partial\left(b_{i} p\right)}{\partial x_{i}}(x)+\frac{1}{2} \sum_{i, j=1}^{n} \frac{\partial^{2}\left(a_{i, j} p\right)}{\partial x_{i} \partial x_{j}}(x)+\int_{0}^{x_{1}} \cdots \int_{0}^{x_{n}} \Phi(\xi) k(\xi, x-\xi) \mathrm{d} \xi-\Phi(x) .
$$

Proof. From Lemma 1, and denoting by $\langle\cdot, \cdot\rangle$ the usual inner product in $\mathbb{R}^{n}$, by considering $f(x)=\exp \{-\langle v, x\rangle\}$, with $v=\left(v_{1}, \ldots, v_{n}\right)$ and $v_{i}>0$ for all $i$, which belongs to $\mathcal{C}_{b}^{2}\left(\mathbb{R}_{+}^{n}\right)$, we obtain

$$
\begin{aligned}
0= & \frac{1}{2} \sum_{i=1}^{n} \frac{v_{i} \mathcal{L}_{n}\left[a_{i, i} p\right](v)-\mathcal{L}_{n-1}\left[a_{i, i}\left(0_{i}\right) p\left(0_{i}^{+}\right)\right]\left(v_{\neq i}\right)}{\prod_{k=1, k \neq i}^{n} v_{k}}+\frac{\mathcal{L}_{n}[h](v)}{\prod_{k=1}^{n} v_{k}}-\frac{\mathcal{L}_{n}[\Phi](v)}{\prod_{k=1}^{n} v_{k}} \\
& -\sum_{i=1}^{n} \frac{\mathcal{L}_{n}\left[b_{i} p\right](v)}{\prod_{k=1, k \neq i}^{n} v_{k}}-\frac{1}{2} \sum_{\substack{i, j=1 \\
i \neq j}}^{n} \frac{R_{i, j}}{R_{j, j}} \frac{\mathcal{L}_{n-1}\left[a_{j, j}\left(0_{j}\right) p\left(0_{j}^{+}\right)\right]\left(v_{\neq j}\right)}{\prod_{k=1, k \neq i, j}^{n} v_{k}} \frac{1}{v_{j}} \\
& +\frac{1}{2} \sum_{\substack{i, j=1 \\
i \neq j}}^{n} \frac{\mathcal{L}_{n}\left[a_{i, j} p\right](v)}{\prod_{k=1, k \neq i, j}^{n} v_{k}},
\end{aligned}
$$

where, for all $x \in \mathbb{R}_{+}^{n}, h(x):=\int_{0}^{x_{1}} \cdots \int_{0}^{x_{n}} \Phi(\xi) k(\xi, x-\xi) \mathrm{d} \xi$. By making the $n$-dimensional inverse Laplace transform of the equation above, and applying $\partial^{n} / \partial x_{1} \cdots \partial x_{n}$ to the equation that results, the corollary follows.

\section{Stationary equations for the separable case}

In this section, we specialize all the previous results to the case where the stationary density $p$ is separable - i.e. it can be expressed as the product of its one-dimensional marginals - and obtain some new ones. These results will lead us to necessary and sufficient conditions for this factorization to be possible, as will be stated in Theorem 1 of Section 4.

From now on, we will assume, in addition to all the assumptions already stated, the following.

1. For all $i$ and $j$ and for all $x \in \mathbb{R}_{+}^{n}, b_{i}(x)=b_{i}\left(x_{i}\right)$ and $a_{i, j}(x)=a_{i, j}\left(x_{i}, x_{j}\right)$. Note that the latter relation holds if, for example, $\sigma_{i, j}(x)=\sigma_{i, j}\left(x_{i}\right)$ for all $i$ and $j$ and for all $x \in \mathbb{R}_{+}^{n}$.

2. For all $x \in \mathbb{R}_{+}^{n}$, the density $k(x, z)$ is separable, taking the form $\prod_{i=1}^{n} k_{i}\left(x_{i}, z_{i}\right)$. That is, for all $x \in \mathbb{R}_{+}^{n}$, the density $k(x, z)$ is written as the product of its one-dimensional marginals and, in addition, its $i$ th one-dimensional marginal depends on the parameter $x$ only through its $i$ th coordinate $x_{i}$, for all $i$.

3. The intensity $\lambda$ is independent of $\left(X_{t-}\right)$ and takes the form $\sum_{i=1}^{n} \lambda_{i}$, where, for all $i, \lambda_{i}$ is the intensity associated with the jumps in the $i$ th coordinate of $\left(X_{t}\right)$, and the $\lambda_{i}$ are independent. 
Remark 1. Note that, since the jumps associated with different coordinates of $\left(X_{t}\right)$ are independent, no two (or more) of its coordinates will jump simultaneously at any given time $t$, P-a.s. Since, furthermore, $\lambda_{j}$ is independent of $\left(X_{t-}^{j}\right)$ and $a_{j, j}\left(0_{j}\right)=a_{j, j}(0)$ for all $j$, equations (2) in Lemma 1 and (4) in Corollary 1 now take the form

$$
\begin{aligned}
0= & \int_{\mathbb{R}_{+}^{n}} \mathcal{T} f(x) p(x) \mathrm{d} x+\frac{1}{2} \sum_{i, j=1}^{n} \frac{R_{i, j}}{R_{j, j}} a_{j, j}(0) \int_{\mathbb{R}_{+}^{n-1}} \frac{\partial f}{\partial x_{i}}\left(0_{j}\right) p\left(0_{j}^{+}\right) \mathrm{d} x_{\neq j} \\
& +\sum_{i=1}^{n} \mathrm{E}\left[\lambda_{i}\right] \int_{\mathbb{R}_{+}^{n+1}}\left\{f\left(x+z_{i}\right)-f(x)\right\} p(x) k_{i}\left(x_{i}, z_{i}\right) \mathrm{d} z_{i} \mathrm{~d} x
\end{aligned}
$$

and

$$
\begin{aligned}
0= & -\sum_{i=1}^{n} \frac{\partial\left(b_{i} p\right)}{\partial x_{i}}(x)+\frac{1}{2} \sum_{i, j=1}^{n} \frac{\partial^{2}\left(a_{i, j} p\right)}{\partial x_{i} \partial x_{j}}(x) \\
& +\sum_{i=1}^{n} \mathrm{E}\left[\lambda_{i}\right] \int_{0}^{x_{i}} p\left(\xi_{i}, x_{\neq i}\right) k_{i}\left(\xi_{i}, x_{i}-\xi_{i}\right) \mathrm{d} \xi_{i}-p(x),
\end{aligned}
$$

respectively, where, for all $i, \mathrm{E}\left[\lambda_{i}\right]$ in (5) and (6) denotes the expected value of the intensity $\lambda_{i}$, and $p\left(\xi_{i}, x_{\neq i}\right)$ in (6) denotes $p\left(x_{1}, \ldots, x_{i-1}, \xi_{i}, x_{i+1}, \ldots, x_{n}\right)$, i.e. the stationary density $p$ when its $i$ th coordinate $x_{i}$ has been replaced by the integration variable $\xi_{i}$. Moreover, (5) holds for all $f \in \mathcal{C}_{b}^{2}\left(\mathbb{R}_{+}^{n}\right)$.

Remark 2. Note that, if $\tilde{p}$ is another density in $\mathbb{R}_{+}^{n}$ satisfying (5) for all $f \in \mathcal{C}_{b}^{2}\left(\mathbb{R}_{+}^{n}\right)$, then $\tilde{p}(x)=p(x)$ for almost every $x \in \mathbb{R}_{+}^{n}$. Moreover, this conclusion still holds if $\tilde{p}$ is another density in $\mathbb{R}_{+}^{n}$ satisfying (5) for all $f=\prod_{i=1}^{n} f_{i}$, where, for all $i, f_{i}$ is any function in $\mathcal{C}_{b}^{2}\left(\mathbb{R}_{+}\right)$.

We now define the operators $\mathcal{T}_{i}: \mathcal{C}^{2}\left(\mathbb{R}_{+}\right) \rightarrow \mathcal{C}\left(\mathbb{R}_{+}\right)$for all $i$, as follows:

$$
\mathcal{T}_{i} f(x):=b_{i}\left(x_{i}\right) \frac{\mathrm{d} f}{\mathrm{~d} x_{i}}\left(x_{i}\right)+\frac{1}{2} a_{i, i}\left(x_{i}\right) \frac{\mathrm{d}^{2} f}{\mathrm{~d} x_{i}^{2}}\left(x_{i}\right) .
$$

Corollary 2. Assume that the stationary density $p$ is separable, i.e. that for almost every $x \in \mathbb{R}_{+}^{n}, p(x)=\prod_{i=1}^{n} p_{i}\left(x_{i}\right)$, where $p_{i}$ is the $i$ th one-dimensional marginal density extracted from $p$, for all $i$. Then $p_{i}$ satisfies the following relationship, for all $i$ and all $f \in \mathcal{C}_{b}^{2}\left(\mathbb{R}_{+}\right)$:

$$
\begin{aligned}
0= & \frac{1}{2} \frac{\mathrm{d} f}{\mathrm{~d} x_{i}}(0) a_{i, i}(0) p_{i}\left(0^{+}\right)+\mathrm{E}\left[\lambda_{i}\right] \int_{\mathbb{R}_{+}^{2}}\left\{f\left(x_{i}+z_{i}\right)-f\left(x_{i}\right)\right\} p_{i}\left(x_{i}\right) k_{i}\left(x_{i}, z_{i}\right) \mathrm{d} z_{i} \mathrm{~d} x_{i} \\
& +\int_{\mathbb{R}_{+}} \tau_{i} f\left(x_{i}\right) p_{i}\left(x_{i}\right) \mathrm{d} x_{i}+\frac{1}{2} \int_{\mathbb{R}_{+}} \frac{\mathrm{d} f}{\mathrm{~d} x_{i}}\left(x_{i}\right) p_{i}\left(x_{i}\right) \mathrm{d} x_{i} \sum_{\substack{j=1 \\
j \neq i}}^{n} \frac{R_{i, j}}{R_{j, j}} a_{j, j}(0) p_{j}\left(0^{+}\right) .
\end{aligned}
$$

Proof. The proof follows in a straightforward manner from (5), by taking $f\left(x_{i}\right) \in \mathcal{C}_{b}^{2}\left(\mathbb{R}_{+}\right)$ for each $i$ and using the product-form of the stationary density $p$. 
Lemma 2. Under the same assumptions as in Corollary 2, for all $i$ we have

$$
-\frac{1}{2} \sum_{j=1}^{n} \frac{R_{i, j}}{R_{j, j}} a_{j, j}(0) p_{j}\left(0^{+}\right)=\int_{\mathbb{R}_{+}} b_{i}\left(x_{i}\right) p_{i}\left(x_{i}\right) \mathrm{d} x_{i}+\mathrm{E}\left[\lambda_{i}\right] \int_{\mathbb{R}_{+}^{2}} z_{i} p_{i}\left(x_{i}\right) k_{i}\left(x_{i}, z_{i}\right) \mathrm{d} z_{i} \mathrm{~d} x_{i} .
$$

Proof. First, for each $X_{i}$, we apply the Meyer-Itô formula [11, p. 167, Theorem 51] with convex function $f\left(x_{i}\right)=\left(x_{i}\right)^{+}=\max \left\{0, x_{i}\right\}$, and use (1). Then, in the stationary regime, by taking expectations and using the fact that the stochastic integrals involved are centred, continuous local martingales, together with conditions (c2) and (c4), Fubini's theorem, and [10, Theorem 3.2 and Corollary 2.1], we find that

$$
\begin{aligned}
& -\frac{1}{2} \int_{\mathbb{R}_{+}} \mathbf{1}_{\left\{x_{i}>0\right\}} p_{i}\left(x_{i}\right) \mathrm{d} x_{i} \sum_{\substack{j=1 \\
j \neq i}}^{n} \frac{R_{i, j}}{R_{j, j}} a_{j, j}(0) p_{j}\left(0^{+}\right) \\
& \quad=\frac{1}{2} a_{i, i}(0) p_{i}\left(0^{+}\right)+\int_{\mathbb{R}_{+}} \mathbf{1}_{\left\{x_{i}>0\right\}} b_{i}\left(x_{i}\right) p_{i}\left(x_{i}\right) \mathrm{d} x_{i}+\mathrm{E}\left[\lambda_{i}\right] \int_{\mathbb{R}_{+}^{2}} z_{i} p_{i}\left(x_{i}\right) k_{i}\left(x_{i}, z_{i}\right) \mathrm{d} z_{i} \mathrm{~d} x_{i},
\end{aligned}
$$

where $\mathbf{1}_{\{\cdot\}}$ denotes the usual indicator function of the event in braces. The lemma now follows.

Remark 3. Note that, since $R$ is nonsingular, from Lemma 2 we obtain that $p_{i}\left(0^{+}\right)>0$ for all $i$ if and only if $R^{-1} \Lambda<0$ (componentwise), where $\Lambda$ is the column vector whose $i$ th entry is given by $\int_{\mathbb{R}_{+}} b_{i}\left(x_{i}\right) p_{i}\left(x_{i}\right) \mathrm{d} x_{i}+\mathrm{E}\left[\lambda_{i}\right] \int_{\mathbb{R}_{+}^{2}} z_{i} p_{i}\left(x_{i}\right) k_{i}\left(x_{i}, z_{i}\right) \mathrm{d} z_{i} \mathrm{~d} x_{i}$, i.e. if and only if the net drift in each dimension is strictly negative. This gives us a stability condition, as will be seen in Section 5.

Corollary 3. Under the same assumptions as in Corollary 2, for all $i$ and all $f \in \mathcal{C}_{b}^{2}\left(\mathbb{R}_{+}\right)$, $p_{i}$ satisfies

$$
\begin{gathered}
0=\int_{\mathbb{R}_{+}} \mathcal{T}_{i} f\left(x_{i}\right) p_{i}\left(x_{i}\right) \mathrm{d} x_{i}+\mathrm{E}\left[\lambda_{i}\right] \int_{\mathbb{R}_{+}^{2}}\left\{f\left(x_{i}+z_{i}\right)-f\left(x_{i}\right)\right\} p_{i}\left(x_{i}\right) k_{i}\left(x_{i}, z_{i}\right) \mathrm{d} z_{i} \mathrm{~d} x_{i} \\
-\int_{\mathbb{R}_{+}} \frac{\mathrm{d} f}{\mathrm{~d} x_{i}}\left(x_{i}\right) p_{i}\left(x_{i}\right) \mathrm{d} x_{i}\left\{\frac{1}{2} a_{i, i}(0) p_{i}\left(0^{+}\right)+\int_{\mathbb{R}_{+}} b_{i}\left(x_{i}\right) p_{i}\left(x_{i}\right) \mathrm{d} x_{i}\right. \\
\left.+\mathrm{E}\left[\lambda_{i}\right] \int_{\mathbb{R}_{+}^{2}} z_{i} p_{i}\left(x_{i}\right) k_{i}\left(x_{i}, z_{i}\right) \mathrm{d} z_{i} \mathrm{~d} x_{i}\right\} \\
+\frac{1}{2} \frac{\mathrm{d} f}{\mathrm{~d} x_{i}}(0) a_{i, i}(0) p_{i}\left(0^{+}\right) .
\end{gathered}
$$

Proof. The proof follows in a straightforward manner, by combining the results of Corollary 2 and Lemma 2.

Remark 4. Note that, as in Remark 2 for $p$, Corollaries 2 and 3 uniquely characterize each one-dimensional marginal density $p_{i}$ extracted from $p$ (almost everywhere, and in the separable setting, of course).

Lemma 3. Under the same assumptions as in Corollary 2, and if, for all $i, p_{i}$ is continuous in $\mathbb{R}_{+}$and $a_{i, i} p_{i} \in \mathcal{C}^{1}\left(\mathbb{R}_{+}\right)$such that the Laplace transform of $\mathrm{d}\left(a_{i, i} p_{i}\right) / \mathrm{d} x_{i}$ exists, 
$p_{i}$ satisfies (9), for all $i$ and all $f \in \mathcal{C}_{b}^{2}\left(\mathbb{R}_{+}\right)$, if and only if it satisfies

$$
\begin{aligned}
0= & \frac{1}{2} \frac{\mathrm{d}\left(a_{i, i} p_{i}\right)}{\mathrm{d} x_{i}}\left(x_{i}\right)+\mathrm{E}\left[\lambda_{i}\right]\left\{\int_{0}^{x_{i}} \int_{0}^{u} p_{i}(\xi) k_{i}(\xi, u-\xi) \mathrm{d} \xi \mathrm{d} u-\int_{0}^{x_{i}} p_{i}(\xi) \mathrm{d} \xi\right\} \\
& +p_{i}\left(x_{i}\right)\left\{\int_{\mathbb{R}_{+}} b_{i}(\xi) p_{i}(\xi) \mathrm{d} \xi-b_{i}\left(x_{i}\right)+\frac{1}{2} a_{i, i}(0) p_{i}(0)\right. \\
& \left.+\mathrm{E}\left[\lambda_{i}\right] \int_{\mathbb{R}_{+}^{2}} z_{i} p_{i}(\xi) k_{i}\left(\xi, z_{i}\right) \mathrm{d} z_{i} \mathrm{~d} \xi\right\} \text { for all } x_{i} \in \mathbb{R}_{+} .
\end{aligned}
$$

Proof. The 'only if' part follows in a straightforward manner from Corollary 3, by considering, for each $i, f\left(x_{i}\right)=\exp \left\{-v_{i} x_{i}\right\}$ with $v_{i}>0$, which belongs to $\mathcal{C}_{b}^{2}\left(\mathbb{R}_{+}\right)$, and then taking the inverse of the resulting Laplace transform. For the 'if' part, consider, for each $i$, $f\left(x_{i}\right) \in \mathcal{C}_{b}^{2}\left(\mathbb{R}_{+}\right)$. Then, by multiplying (10) by $-\mathrm{d} f / \mathrm{d} x_{i}$, integrating over $\mathbb{R}_{+}$, and using the fact that, by condition (c3) with $i=j, a_{i, i}\left(x_{i}\right) p_{i}\left(x_{i}\right) \rightarrow 0$ as $x_{i} \rightarrow \infty$, we obtain (9). The lemma is now proved.

Remark 5. Assuming that $p_{i}, b_{i} p_{i} \in \mathcal{C}^{1}\left(\mathbb{R}_{+}\right)$and $a_{i, i} p_{i} \in \mathcal{C}^{2}\left(\mathbb{R}_{+}\right)$for all $i$, and that the Laplace transform of $\mathrm{d}\left(a_{i, i} p_{i}\right) / \mathrm{d} x_{i}$ exists, by applying $\mathrm{d} / \mathrm{d} x_{i}$ to (10) we obtain the FokkerPlanck equation for each coordinate, when we work in the stationary regime with a product-form density. That is,

$$
\begin{aligned}
0= & \frac{\mathrm{d} p_{i}}{\mathrm{~d} x_{i}}\left(x_{i}\right)\left\{\int_{\mathbb{R}_{+}} b_{i}(\xi) p_{i}(\xi) \mathrm{d} \xi+\frac{1}{2} a_{i, i}(0) p_{i}(0)+\mathrm{E}\left[\lambda_{i}\right] \int_{\mathbb{R}_{+}^{2}} z_{i} p_{i}(\xi) k_{i}\left(\xi, z_{i}\right) \mathrm{d} z_{i} \mathrm{~d} \xi\right\} \\
& -\mathrm{E}\left[\lambda_{i}\right] p_{i}\left(x_{i}\right)-\frac{\mathrm{d}\left(b_{i} p_{i}\right)}{\mathrm{d} x_{i}}\left(x_{i}\right)+\frac{1}{2} \frac{\mathrm{d}^{2}\left(a_{i, i} p_{i}\right)}{\mathrm{d} x_{i}^{2}}\left(x_{i}\right)+\mathrm{E}\left[\lambda_{i}\right] \int_{0}^{x_{i}} p_{i}(\xi) k_{i}\left(\xi, x_{i}-\xi\right) \mathrm{d} \xi .
\end{aligned}
$$

Remark 6. Note that if, for all $i$ and for all $x_{i} \in \mathbb{R}_{+}, b_{i}\left(x_{i}\right)=b_{i}$ (a constant) and $k_{i}\left(x_{i}, z_{i}\right)=$ $k_{i}\left(z_{i}\right)$ (independent of $x_{i}$ ), then (10) has no anticipative terms (in space). It can therefore be solved numerically using, in this case, the known boundary conditions $\left\{p_{i}(0)\right\}_{i=1}^{n}$ given by (8) (also see Remark 3).

\section{The main result: necessary and sufficient conditions}

Using the results of the previous sections, we are now in position to state and prove the main result of the paper, concerning necessary and sufficient conditions for product-form distributions in the stationary regime, assuming that this regime and its corresponding density $p$, satisfying conditions (c1) to (c4), exist. (Note that we have not shown that the existence of a solution to (5) is sufficient for the existence of a stationary regime.)

Theorem 1. Let $\left\{p_{i}\right\}_{i=1}^{n}$ be the family of one-dimensional marginals extracted from the stationary density $p$. Then $p$ is separable, i.e. $p(x)=\prod_{i=1}^{n} p_{i}\left(x_{i}\right)$ for almost every $x \in \mathbb{R}_{+}^{n}$, if and only if, for all $i$ and $j, i \neq j$, we have

$$
a_{i, j}\left(x_{i}, x_{j}\right)=g_{i, j}\left(x_{i}\right)+g_{j, i}\left(x_{j}\right)
$$

for $\left(F_{i} \times F_{j}\right)$-almost-every $\left(x_{i}, x_{j}\right) \in \mathbb{R}_{+}^{2}$. Here, for all $k, F_{k}$ denotes the law in $\mathbb{R}_{+}$associated with $p_{k}$ and, for all $k$ and $l, k \neq l$, the entries of the tuple $\left(g_{k, l}, g_{l, k}\right)$ are defined, $F_{k} \times F_{l}$ 
almost everywhere, by

$$
g_{k, l}\left(x_{k}\right):=\frac{R_{l, k}}{2 R_{k, k}} \frac{a_{k, k}(0) p_{k}\left(0^{+}\right)}{p_{k}\left(x_{k}\right)} \int_{x_{k}}^{\infty} p_{k}(\xi) \mathrm{d} \xi
$$

and, for $g_{l, k}\left(x_{l}\right)$, the expression obtained by interchanging $k$ and $l$ in this expression.

Proof. We assume that $p$ is separable. Then, using (7) and (5) with $f=\prod_{i=1}^{n} f_{i}$, where, for all $i, f_{i}$ is any function in $\mathcal{C}_{b}^{2}\left(\mathbb{R}_{+}\right)$, we obtain

$$
0=\sum_{\substack{i, j=1 \\ i \neq j}}^{n} \Theta_{i, j}\left(f_{i}, f_{j}\right) \prod_{\substack{k=1 \\ k \neq i, j}}^{n} \int_{\mathbb{R}_{+}} f_{k}\left(x_{k}\right) p_{k}\left(x_{k}\right) \mathrm{d} x_{k}
$$

where

$$
\Theta_{i, j}\left(f_{i}, f_{j}\right):=\Gamma_{i, j}\left(f_{i}, f_{j}\right)+\int_{\mathbb{R}_{+}^{2}} \frac{\mathrm{d} f_{i}}{\mathrm{~d} x_{i}}\left(x_{i}\right) \frac{\mathrm{d} f_{j}}{\mathrm{~d} x_{j}}\left(x_{j}\right) a_{i, j}\left(x_{i}, x_{j}\right) p_{i}\left(x_{i}\right) p_{j}\left(x_{j}\right) \mathrm{d} x_{i} \mathrm{~d} x_{j}
$$

and

$$
\Gamma_{i, j}\left(f_{i}, f_{j}\right):=\frac{R_{i, j}}{R_{j, j}} a_{j, j}(0) p_{j}\left(0^{+}\right) \int_{\mathbb{R}_{+}} \frac{\mathrm{d} f_{i}}{\mathrm{~d} x_{i}}\left(x_{i}\right) p_{i}\left(x_{i}\right) \mathrm{d} x_{i}\left\{f_{j}(0)-\int_{\mathbb{R}_{+}} f_{j}\left(x_{j}\right) p_{j}\left(x_{j}\right) \mathrm{d} x_{j}\right\} .
$$

Now, (13) holds for any $f$ of the considered form if and only if $\Theta_{i, j}\left(f_{i}, f_{j}\right)+\Theta_{j, i}\left(f_{j}, f_{i}\right)=0$ for all $i$ and $j, i \neq j$, and all $f_{i}, f_{j} \in \mathcal{C}_{b}^{2}\left(\mathbb{R}_{+}\right)$. Thus, for $i \neq j, a_{i, j}$ must be such that, for all $f_{i}, f_{j} \in \mathcal{C}_{b}^{2}\left(\mathbb{R}_{+}\right)$,

$$
2 \int_{\mathbb{R}_{+}^{2}} \frac{\mathrm{d} f_{i}}{\mathrm{~d} x_{i}}\left(x_{i}\right) \frac{\mathrm{d} f_{j}}{\mathrm{~d} x_{j}}\left(x_{j}\right) a_{i, j}\left(x_{i}, x_{j}\right) p_{i}\left(x_{i}\right) p_{j}\left(x_{j}\right) \mathrm{d} x_{i} \mathrm{~d} x_{j}=\Gamma_{i, j}\left(f_{i}, f_{j}\right)+\Gamma_{j, i}\left(f_{j}, f_{i}\right),
$$

where we have used the fact that the diffusion matrix $a$ is symmetric. Thinking of the above expression as an equation for $a_{i, j}$, with $i \neq j$, we immediately obtain uniqueness in the $\left(F_{i} \times F_{j}\right)$-almost-everywhere sense. Therefore, for $i \neq j$, we may look for a solution of the form $a_{i, j}\left(x_{i}, x_{j}\right)=g_{i, j}\left(x_{i}\right)+g_{j, i}\left(x_{j}\right)$. Inserting this in (14), and considering $f_{k}\left(x_{k}\right)=\exp \left\{-v_{k} x_{k}\right\}$ with $v_{k}>0, k=i, j$, which belong to $\mathcal{C}_{b}^{2}\left(\mathbb{R}_{+}\right)$, we obtain

$$
\begin{aligned}
\mathscr{L}\left[g_{i, j} p_{i}\right]\left(v_{i}\right) \mathcal{L}\left[p_{j}\right]\left(v_{j}\right)+\mathcal{L}\left[g_{j, i} p_{j}\right]\left(v_{j}\right) \mathcal{L}\left[p_{i}\right]\left(v_{i}\right) \\
=\frac{R_{i, j}}{2 R_{j, j}} a_{j, j}(0) p_{j}\left(0^{+}\right)\left\{\frac{1}{v_{j}}-\frac{\mathcal{L}\left[p_{j}\right]\left(v_{j}\right)}{v_{j}}\right\} \mathcal{L}\left[p_{i}\right]\left(v_{i}\right) \\
\quad+\frac{R_{j, i}}{2 R_{i, i}} a_{i, i}(0) p_{i}\left(0^{+}\right)\left\{\frac{1}{v_{i}}-\frac{\mathcal{L}\left[p_{i}\right]\left(v_{i}\right)}{v_{i}}\right\} \mathcal{L}\left[p_{j}\right]\left(v_{j}\right) .
\end{aligned}
$$

Since the above equation must hold for all $v_{i}, v_{j}>0$, we conclude that

$$
\mathcal{L}\left[g_{i, j} p_{i}\right]\left(v_{i}\right)=\frac{R_{j, i}}{2 R_{i, i}} a_{i, i}(0) p_{i}\left(0^{+}\right)\left\{\frac{1}{v_{i}}-\frac{\mathcal{L}\left[p_{i}\right]\left(v_{i}\right)}{v_{i}}\right\},
$$

with the corresponding expression for $\mathcal{L}\left[g_{j, i} p_{j}\right]\left(v_{j}\right)$ gained by interchanging $i$ and $j$. Hence, (11) and (12) now follow. For the converse, assume that for all $i$ and $j, i \neq j, a_{i, j}\left(x_{i}, x_{j}\right)$ 
satisfies (11) for $\left(F_{i} \times F_{j}\right)$-almost-every $\left(x_{i}, x_{j}\right) \in \mathbb{R}_{+}^{2}$, with $g_{i, j}$ and $g_{j, i}$ as in (12). Since, for all $i$ and all $f_{i} \in \mathcal{C}_{b}^{2}\left(\mathbb{R}_{+}\right)$, we have

$$
\begin{aligned}
\int_{\mathbb{R}_{+}} \frac{\mathrm{d} f_{i}}{\mathrm{~d} x_{i}}\left(x_{i}\right)\left(\int_{x_{i}}^{\infty} p_{i}(\xi) \mathrm{d} \xi\right) \mathrm{d} x_{i}= & \lim _{x_{i} \rightarrow \infty} f_{i}\left(x_{i}\right) \int_{x_{i}}^{\infty} p_{i}(\xi) \mathrm{d} \xi-f_{i}(0) \\
& +\int_{\mathbb{R}_{+}} f_{i}\left(x_{i}\right) p_{i}\left(x_{i}\right) \mathrm{d} x_{i} \\
= & -f_{i}(0)+\int_{\mathbb{R}_{+}} f_{i}\left(x_{i}\right) p_{i}\left(x_{i}\right) \mathrm{d} x_{i},
\end{aligned}
$$

it follows that $\Theta_{i, j}\left(f_{i}, f_{j}\right)+\Theta_{j, i}\left(f_{j}, f_{i}\right)=0$ for all $i$ and $j, i \neq j$, and all $f_{i}, f_{j} \in \mathcal{C}_{b}^{2}\left(\mathbb{R}_{+}\right)$. Therefore, (5) is satisfied with $\prod_{i=1}^{n} p_{i}$ in place of $p$ for all $f=\prod_{i=1}^{n} f_{i}$, where, for all $i, f_{i}$ is any function in $\mathcal{C}_{b}^{2}\left(\mathbb{R}_{+}\right)$. The theorem now follows (see Remark 2).

Remark 7. Note that, in the statement of Theorem 1, no assumptions regarding the existence of derivatives involving $b, a$, or $p$ are needed.

Remark 8. In the case where there are no jumps and the drift vector $b(\cdot)$ is constant, by considering $f_{i}\left(x_{i}\right)=\exp \left\{-v_{i} x_{i}\right\} \in \mathcal{C}_{b}^{2}\left(\mathbb{R}_{+}\right)\left(v_{i}>0\right)$, for each $i$, from (9) we obtain

$$
a_{i, i}(0) p_{i}\left(0^{+}\right)\left\{\frac{1}{v_{i}}-\frac{\mathcal{L}\left[p_{i}\right]\left(v_{i}\right)}{v_{i}}\right\}=\mathcal{L}\left[a_{i, i} p_{i}\right]\left(v_{i}\right)
$$

and, therefore, using (15) the separability condition can be written, in this case, as

$$
a_{i, j}\left(x_{i}, x_{j}\right)=\frac{R_{j, i}}{2 R_{i, i}} a_{i, i}\left(x_{i}\right)+\frac{R_{i, j}}{2 R_{j, j}} a_{j, j}\left(x_{j}\right)
$$

for all $i$ and $j, i \neq j$, and for $\left(F_{i} \times F_{j}\right.$ )-almost-every $\left(x_{i}, x_{j}\right) \in \mathbb{R}_{+}^{2}$ (as will be seen in Subsection 5.1.1, under the conditions therein, for all $\left.\left(x_{i}, x_{j}\right) \in \mathbb{R}_{+}^{2}\right)$. Note that this generalizes the separability condition stated in [4] for the simpler case of SRBM, where the entries of $b$ and $a$ do not depend on $x$ and there are no jumps. Finally, note that from (16), condition (c3) is satisfied for all $i$ and $j$, in the separable case, once satisfied for all $i=j$.

From Theorem 1, we conclude that product-form stationary distributions are only possible under very special conditions. In fact, we have the following negative result.

Corollary 4. If the diffusion matrix a has constant off-diagonal elements, then a product-form stationary density is not possible unless either the jumps in each coordinate are identically null and the drift $b$, as well as the diagonal elements of $a$, are constant; or both $a$ and the reflection matrix $R$ are diagonal.

Proof. Let $\left\{p_{i}\right\}_{i=1}^{n}$ be the family of one-dimensional marginals extracted from the stationary density $p$, and assume that for all $i$ and $j, i \neq j, a_{i, j}\left(x_{i}, x_{j}\right)=a_{i, j}$ (a constant). Then, from Theorem 1 , a product-form stationary density is only possible in the following two cases. The first case is when each $p_{i}$ is such that

$$
\frac{1}{p_{i}\left(x_{i}\right)} \int_{x_{i}}^{\infty} p_{i}(\xi) \mathrm{d} \xi=\frac{1}{p_{i}\left(0^{+}\right)} \quad \text { for all } x_{i} \in \mathbb{R}_{+} .
$$

However, then $p_{i}$ is absolutely continuous for all $i$, thus continuous and, therefore, from the same expression, differentiable. Hence, $p_{i}\left(x_{i}\right)=p_{i}\left(0^{+}\right) \exp \left\{-p_{i}\left(0^{+}\right) x_{i}\right\}$ for all $i$ and all 
$x_{i} \in \mathbb{R}_{+}$, i.e. each $p_{i}$ corresponds to an exponential density with parameter $p_{i}\left(0^{+}\right)=p_{i}(0)$, in which case, from Corollary 3 , the jumps in each coordinate must be identically null and the drift $b$, as well as the diagonal elements of $a$, must be constant. Note that this case corresponds to SRBM. The second case is when, for all $i$ and $j, i \neq j, a_{i, j}=0$ (i.e. $a$ is diagonal) and either $R_{i, j}=0$ or $p_{j}\left(0^{+}\right)=0$. But then, from Lemma 2, we conclude that, for all $i$,

$$
0=\frac{1}{2} a_{i, i}(0) p_{i}\left(0^{+}\right)+\int_{\mathbb{R}_{+}} b_{i}\left(x_{i}\right) p_{i}\left(x_{i}\right) \mathrm{d} x_{i}+\mathrm{E}\left[\lambda_{i}\right] \int_{\mathbb{R}_{+}^{2}} z_{i} p_{i}\left(x_{i}\right) k_{i}\left(x_{i}, z_{i}\right) \mathrm{d} z_{i} \mathrm{~d} x_{i}
$$

and, therefore, from Corollary 3 , if $p_{i}\left(0^{+}\right)=0$ for some $i$ then $p_{i}\left(x_{i}\right)=0$ for almost every $x_{i} \in \mathbb{R}_{+}$, which cannot be. Thus, we conclude that $R$ must be diagonal as well. The corollary is now proved.

Remark 9. Note that Corollary 4 generalizes the negative results regarding product-form stationary distributions shown for the case of $n$-dimensional reflected Lévy processes in [8].

\section{Some examples}

We conclude the paper with a presentation of some examples where explicit computations are possible.

\subsection{Continuous case}

We first discuss some examples in the continuous case, i.e. in the absence of jumps. Then, assuming that the hypotheses of Lemma 3 are satisfied and that, in addition, for all $i$ and all $x_{i} \in \mathbb{R}_{+}, a_{i, i}\left(x_{i}\right)>0$, from (10) we find that

$$
p_{i}\left(x_{i}\right)=\frac{a_{i, i}(0) p_{i}(0)}{a_{i, i}\left(x_{i}\right)} \exp \left\{-2 \int_{0}^{x_{i}} \frac{\mathrm{E}\left[b_{i}\right]-b_{i}(\xi)+\frac{1}{2} a_{i, i}(0) p_{i}(0)}{a_{i, i}(\xi)} \mathrm{d} \xi\right\}
$$

for all $i$ and all $x_{i} \in \mathbb{R}_{+}$, where $\mathrm{E}\left[b_{i}\right]=\int_{\mathbb{R}_{+}} b_{i}(\zeta) p_{i}(\zeta) \mathrm{d} \zeta$. We can find all the unknowns involved in (17) in the following two cases.

5.1.1. Constant drift vector $b$. In this case, (17) reduces to

$$
p_{i}\left(x_{i}\right)=\frac{a_{i, i}(0) p_{i}(0)}{a_{i, i}\left(x_{i}\right)} \exp \left\{-a_{i, i}(0) p_{i}(0) \int_{0}^{x_{i}} \frac{\mathrm{d} \xi}{a_{i, i}(\xi)}\right\} .
$$

Furthermore, from Lemma 2, we have $R \gamma_{0}=-2 b$, where $b$ is the constant drift vector and $\gamma_{0}$ is the vector whose $i$ th entry is given by $\left(a_{i, i}(0) / R_{i, i}\right) p_{i}(0)$. Then, since $R$ is nonsingular, the boundary conditions $\left\{p_{i}(0)\right\}_{i=1}^{n}$ are uniquely determined by inverting the above system of linear equations. Of course, we require that $\gamma_{0}>0$ (componentwise), which imposes the stability condition $R^{-1} b<0$ (componentwise), i.e. the net drift in each dimension must be strictly negative. Furthermore, if for all $i$,

$$
\int_{\mathbb{R}_{+}} \frac{\mathrm{d} x_{i}}{a_{i, i}\left(x_{i}\right)}=\infty
$$

then each $p_{i}$ integrates to unity. Note that if $a_{i, i}$ is constant, the above condition is trivially satisfied and, from (18), $p_{i}$ corresponds to an exponential density with parameter $p_{i}(0)$. Moreover, note that, from (18),

$$
\frac{\mathrm{d}\left(a_{i, i} p_{i}\right)}{\mathrm{d} x_{i}}\left(x_{i}\right)=-a_{i, i}(0) p_{i}(0) p_{i}\left(x_{i}\right)
$$


and, therefore, that the Laplace transform of $\mathrm{d}\left(a_{i, i} p_{i}\right) / \mathrm{d} x_{i}$ exists for all $i$. In addition, if for all $i$,

$$
\int_{\mathbb{R}_{+}} \exp \left\{-a_{i, i}(0) p_{i}(0) \int_{0}^{x_{i}} \frac{\mathrm{d} \xi}{a_{i, i}(\xi)}\right\} \mathrm{d} x_{i}<\infty,
$$

then condition (c3) is satisfied (in the separable setting) for all $i$ and $j$, since the separability condition in this case reduces to (see Remark 8)

$$
a_{i, j}\left(x_{i}, x_{j}\right)=\frac{R_{j, i}}{2 R_{i, i}} a_{i, i}\left(x_{i}\right)+\frac{R_{i, j}}{2 R_{j, j}} a_{j, j}\left(x_{j}\right)
$$

for all $i$ and $j, i \neq j$, and for $\left(F_{i} \times F_{j}\right)$-almost-every $\left(x_{i}, x_{j}\right) \in \mathbb{R}_{+}^{2}$, i.e. for all $\left(x_{i}, x_{j}\right) \in \mathbb{R}_{+}^{2}$, since the elements of $a$ are continuous. Then, from (18), we conclude that $F_{i}$ is equivalent to the Lebesgue measure in $\mathbb{R}_{+}$for all $i$. Thus, under all the previous requirements, if the stationary setting and its corresponding density $p$, satisfying conditions (c3) and (c4), exist, then

$$
p(x)=\prod_{i=1}^{n} \frac{a_{i, i}(0) p_{i}(0)}{a_{i, i}\left(x_{i}\right)} \exp \left\{-a_{i, i}(0) p_{i}(0) \int_{0}^{x_{i}} \frac{\mathrm{d} \xi}{a_{i, i}(\xi)}\right\}
$$

for almost every $x \in \mathbb{R}_{+}^{n}$. Note that this generalizes the exponential product-form density obtained in the case of SRBM, as shown, for example, in [1] and [4].

5.1.2. Normal reflections. In this case, the reflection matrix $R$ is the identity matrix and, therefore, using Lemma 2, (17) reduces to

$$
p_{i}\left(x_{i}\right)=\frac{a_{i, i}(0) p_{i}(0)}{a_{i, i}\left(x_{i}\right)} \exp \left\{2 \int_{0}^{x_{i}} \frac{b_{i}(\xi)}{a_{i, i}(\xi)} \mathrm{d} \xi\right\} .
$$

From the normalization condition, we obtain

$$
p_{i}(0)=\left\{a_{i, i}(0) \int_{\mathbb{R}_{+}} \frac{\phi_{i}\left(x_{i}\right)}{a_{i, i}\left(x_{i}\right)} \mathrm{d} x_{i}\right\}^{-1},
$$

where

$$
\phi_{i}\left(x_{i}\right):=\exp \left\{2 \int_{0}^{x_{i}} \frac{b_{i}(\xi)}{a_{i, i}(\xi)} \mathrm{d} \xi\right\},
$$

and, therefore, we require that, for all $i$,

$$
\int_{\mathbb{R}_{+}} \frac{\phi_{i}\left(x_{i}\right)}{a_{i, i}\left(x_{i}\right)} \mathrm{d} x_{i}<\infty .
$$

Furthermore, note that for all $i,(8)$ in Lemma 2 is verified if $b_{i}\left(x_{i}\right)<0$ for almost every $x_{i} \in \mathbb{R}_{+}$, and if

$$
\int_{\mathbb{R}_{+}} \frac{b_{i}\left(x_{i}\right)}{a_{i, i}\left(x_{i}\right)} \mathrm{d} x_{i}=-\infty .
$$

Also, note that the above requirements guarantee that condition (c2) is satisfied for all $i$. Moreover, note that, from (19),

$$
\frac{\mathrm{d}\left(a_{i, i} p_{i}\right)}{\mathrm{d} x_{i}}\left(x_{i}\right)=2 b_{i}\left(x_{i}\right) p_{i}\left(x_{i}\right)
$$


and, therefore, under the previous requirements, the Laplace transform of $\mathrm{d}\left(a_{i, i} p_{i}\right) / \mathrm{d} x_{i}$ exists for all $i$. In addition, if, for all $i$,

$$
\int_{\mathbb{R}_{+}} \exp \left\{2 \int_{0}^{x_{i}} \frac{b_{i}(\xi)}{a_{i, i}(\xi)} \mathrm{d} \xi\right\} \mathrm{d} x_{i}<\infty,
$$

then condition (c3) is satisfied (in the separable setting) for all $i$ and $j$, since the separability condition in this case reduces, of course, to

$$
a_{i, j}\left(x_{i}, x_{j}\right)=0
$$

for all $i$ and $j, i \neq j$, and for $\left(F_{i} \times F_{j}\right)$-almost-every $\left(x_{i}, x_{j}\right) \in \mathbb{R}_{+}^{2}$, i.e. for all $\left(x_{i}, x_{j}\right) \in \mathbb{R}_{+}^{2}$, since the elements of $a$ are continuous. Then, from (19), we conclude that $F_{i}$ is equivalent to the Lebesgue measure in $\mathbb{R}_{+}$for all $i$. Thus, under all the previous requirements, if the stationary setting and its corresponding density $p$, satisfying conditions (c2), (c3), and (c4), exist, then

$$
p(x)=\prod_{i=1}^{n} \frac{a_{i, i}(0) p_{i}(0)}{a_{i, i}\left(x_{i}\right)} \exp \left\{2 \int_{0}^{x_{i}} \frac{b_{i}(\xi)}{a_{i, i}(\xi)} \mathrm{d} \xi\right\}
$$

for almost every $x \in \mathbb{R}_{+}^{n}$.

\subsection{Càdlàg case}

We now allow a nonidentically null-jump measure. Then, assuming that, for all $i$ and all $x_{i} \in \mathbb{R}_{+}, b_{i}\left(x_{i}\right)=b_{i}$ (a constant), $a_{i, i}\left(x_{i}\right)=a_{i, i}>0$ (a constant), and $k_{i}\left(x_{i}, z_{i}\right)=k_{i}\left(z_{i}\right)$ (independent of $\left.x_{i}\right)$, by considering $f_{i}\left(x_{i}\right)=\exp \left\{-v_{i} x_{i}\right\} \in \mathcal{C}_{b}^{2}\left(\mathbb{R}_{+}\right)\left(v_{i}>0\right)$ for each $i$, from (9) we find that

$$
\mathcal{L}\left[p_{i}\right]\left(v_{i}\right)=\frac{\frac{1}{2} a_{i, i} p_{i}\left(0^{+}\right)}{\frac{1}{2} a_{i, i} p_{i}\left(0^{+}\right)+\mathrm{E}\left[\lambda_{i}\right] \mathrm{E}\left[k_{i}\right]+\frac{1}{2} a_{i, i} v_{i}+\left(\mathrm{E}\left[\lambda_{i}\right] / v_{i}\right)\left\{\mathcal{L}\left[k_{i}\right]\left(v_{i}\right)-1\right\}},
$$

where $\mathrm{E}\left[k_{i}\right]=\int_{\mathbb{R}_{+}} z_{i} k_{i}\left(z_{i}\right) \mathrm{d} z_{i}$. Of course, we require that $\mathrm{E}\left[\lambda_{i}\right] \mathrm{E}\left[k_{i}\right]<\infty$ for all $i$. Furthermore, from Lemma 2, we have

$$
R \gamma_{0}=-2 \beta,
$$

where $\gamma_{0}$ is as in Subsection 5.1 .1 (but, of course, with $p_{i}(0)$ replaced by $p_{i}\left(0^{+}\right)$for all $i$ ) and $\beta$ is the vector whose $i$ th entry is given by $b_{i}+\mathrm{E}\left[\lambda_{i}\right] \mathrm{E}\left[k_{i}\right]$. Then, since $R$ is nonsingular, the limit boundary conditions $\left\{p_{i}\left(0^{+}\right)\right\}_{i=1}^{n}$ are uniquely determined by inverting the above system of linear equations. Therefore, as in Subsection 5.1.1, we require that $\gamma_{0}>0$ (componentwise), i.e. the net drift in each dimension must be strictly negative. Thus, under the previous requirements, if the stationary setting and its corresponding density $p$, satisfying conditions (c3) and (c4), exist, and if condition (c3) and the separability condition in Theorem 1 are satisfied by the family $\left\{p_{i}\right\}_{i=1}^{n}$ obtained from (20), then

$$
p(x)=\prod_{i=1}^{n} p_{i}\left(x_{i}\right)
$$

for almost every $x \in \mathbb{R}_{+}^{n}$. (Note that condition (c3) is trivially satisfied when $i=j$, since $a$ has constant diagonal elements.) 
5.2.1. Exponentially distributed jumps. As a particular example of the results above, we now consider the case of exponentially distributed jumps, i.e. when $k_{i}$ corresponds to an exponential density with parameter $\theta_{i}>0$, for all $i$. Then, by making the inverse Laplace transform of (20), we obtain, for all $i$ and for almost every $x_{i} \in \mathbb{R}_{+}$,

$$
p_{i}\left(x_{i}\right)=\frac{1}{2} p_{i}\left(0^{+}\right)\left\{q_{i}\left(x_{i}\right)+r_{i}\left(x_{i}\right)\right\}
$$

where

$$
\begin{aligned}
& q_{i}\left(x_{i}\right):=\left(1-\frac{M_{i}}{N_{i}}\right) \exp \left\{-\left(\theta_{i}+M_{i}-N_{i}\right) x_{i}\right\} \\
& r_{i}\left(x_{i}\right):=\left(1+\frac{M_{i}}{N_{i}}\right) \exp \left\{-\left(\theta_{i}+M_{i}+N_{i}\right) x_{i}\right\}
\end{aligned}
$$

and

$$
\begin{aligned}
2 M_{i} & :=p_{i}\left(0^{+}\right)-\theta_{i}+\frac{2 \mathrm{E}\left[\lambda_{i}\right]}{a_{i, i} \theta_{i}}, \\
N_{i} & :=\sqrt{\frac{2 \mathrm{E}\left[\lambda_{i}\right]}{a_{i, i}}+M_{i}^{2}} \geq\left|M_{i}\right| .
\end{aligned}
$$

Then, since

$$
\theta_{i}+M_{i}=\frac{p_{i}\left(0^{+}\right)}{2}+\frac{\theta_{i}}{2}+\frac{\mathrm{E}\left[\lambda_{i}\right]}{a_{i, i} \theta_{i}}
$$

and

$$
N_{i}=\sqrt{\left\{\frac{p_{i}\left(0^{+}\right)}{2}+\frac{\theta_{i}}{2}+\frac{\mathrm{E}\left[\lambda_{i}\right]}{a_{i, i} \theta_{i}}\right\}^{2}-p_{i}\left(0^{+}\right) \theta_{i}}
$$

for all $i,(22)$ gives a valid density in $\mathbb{R}_{+}$as long as $p_{i}\left(0^{+}\right)>0$ (see (21) and the comments that follow it). In addition, since $\int_{\mathbb{R}_{+}} x_{i} p_{i}\left(x_{i}\right) \mathrm{d} x_{i}<\infty$ for all $i$, condition (c3) is satisfied for all $i$ and $j$ in the separable case. Finally, the separability condition is

$$
a_{i, j}\left(x_{i}, x_{j}\right)=g_{i, j}\left(x_{i}\right)+g_{j, i}\left(x_{j}\right)
$$

for all $i$ and $j, i \neq j$, and for all $\left(x_{i}, x_{j}\right) \in \mathbb{R}_{+}^{2}$ since the elements of $a$ are continuous and, from (22), we conclude that $F_{i}$ is equivalent to the Lebesgue measure in $\mathbb{R}_{+}$and that $p_{i}$ can be taken to be continuous and strictly positive, for all $i$. Here, for all $k$ and $l, k \neq l$,

$$
g_{k, l}\left(x_{k}\right)=\frac{R_{l, k}}{2 R_{k, k}} a_{k, k} p_{k}\left(0^{+}\right) \frac{q_{k}\left(x_{k}\right) /\left(\theta_{k}+M_{k}-N_{k}\right)+r_{k}\left(x_{k}\right) /\left(\theta_{k}+M_{k}+N_{k}\right)}{q_{k}\left(x_{k}\right)+r_{k}\left(x_{k}\right)} .
$$

Thus, under all the previous requirements, if the stationary setting and its corresponding density $p$, satisfying conditions (c3) and (c4), exist, then

$$
p(x)=\frac{1}{2} \prod_{i=1}^{n} p_{i}\left(0^{+}\right)\left\{q_{i}\left(x_{i}\right)+r_{i}\left(x_{i}\right)\right\}
$$

for almost every $x \in \mathbb{R}_{+}^{n}$. 


\section{Concluding remarks}

Although, in this paper, we have provided necessary and sufficient conditions for productform densities to exist in the stationary regime, we have not discussed the conditions forthe existence of this regime. However, we believe the conditions derived in Section 5 are the appropriate ones in each of the cases considered there (in the separable setting, of course). We also believe that product-form stationary densities are not possible under any more general dependences of $b$ and $a$ on $x \in \mathbb{R}_{+}^{n}$ than the ones we have considered here, or under a more general, nonseparable structure for the jumps. Moreover, in this paper we have considered the case of positive jumps. However, all the results in the paper are readily generalizable (mutatis mutandis) to the case of both positive and negative jumps, under the additional assumption that $\Pi$ is such that for all $t \in \mathbb{R}_{+}, X_{t-}+\Delta X_{t} \geq 0$ (componentwise) P-a.s., where $\Delta X_{t}:=X_{t}-X_{t-}$. Then $\left(X_{t}\right)$ does not leave the positive orthant due to a jump and, in addition, the regulator (or reflection map) process $\left(Z_{t}\right)$ remains continuous. Of course, condition (c1) is in this case extended to $\int_{\mathbb{R}_{+}^{n} \times \mathbb{R}^{n}}\left|z_{i}\right| \Phi(x) k(x, z) \mathrm{d} z \mathrm{~d} x=\int_{\mathbb{R}_{+}^{n} \times \mathbb{R}}\left|z_{i}\right| \Phi(x) k_{i}\left(x, z_{i}\right) \mathrm{d} z_{i} \mathrm{~d} x<\infty$ for all $i$. Note that models including negative jumps are important in risk theory and in financial scenarios where claims arise at random times.

\section{Acknowledgements}

This research was supported in part by the National Science Foundation grant ANI-0087404 and by a doctoral scholarship (FP) from the University of Chile.

\section{References}

[1] Bardhan, I. (1995). Further applications of a general rate conservation law. Stoch. Process. Appl. 60, $113-130$.

[2] Berman, A. And Plemmons, R. J. (1994). Nonnegative Matrices in the Mathematical Sciences (Classics Appl. Math. 9). Society for Industrial and Applied Mathematics, Philadelphia, PA.

[3] Davis, M. H. A. (1984). Piecewise-deterministic Markov processes: a general class of nondiffusion stochastic models: with discussion. J. R. Statist. Soc. B 46, 353-388.

[4] Harrison, J. AND Williams, R. J. (1987). Brownian models of open queueing networks with homogeneous customer populations. Stochastics 22, 77-115.

[5] JACOD, J. AND Shiryaev, A. N. (1987). Limit Theorems for Stochastic Processes (Fundamental Principles Math. Sci. 288). Springer, Berlin.

[6] Kella, O. (2000). Non-product form of two-dimensional fluid networks with dependent Lévy inputs. J. Appl. Prob. 37, 1117-1122.

[7] Kella, O. And Whitt, W. (1990). Diffusion approximations for queues with server vacations. Adv. Appl. Prob. 22, 706-729.

[8] Konstantopoulos, T., Last, G. and Lin, S.-J. (2004). On a class of Lévy stochastic networks. Queueing Systems 46, 409-437.

[9] Mazumdar, R. R. and Guillemin, F. M. (1996). Forward equations for reflected diffusions with jumps. Appl. Math. Optimization 33, 81-102.

[10] Piera, F., Mazumdar, R. and Guillemin, F. (2004). On the local times and boundary properties of reflected diffusions with jumps in the positive orthant. Submitted.

[11] Protter, P. (1990). Stochastic Integration and Differential Equations: A New Approach (Appl. Math. 21). Springer, Berlin.

[12] Reiman, M. I. and Williams, R. J. (1988). A boundary property of semimartingale reflecting Brownian motions. Prob. Theory Relat. Fields 77, 87-97.

[13] Shen, X., Chen, H., DaI, J. G. AND DaI, W. (2002). The finite element method for computing the stationary distribution of an SRBM in a hypercube with applications to finite buffer queueing networks. Queueing Systems 42, 33-62.

[14] Taylor, L. M. and Williams, R. J. (1993). Existence and uniqueness of semimartingale reflecting Brownian motions in an orthant. Prob. Theory Relat. Fields 96, 283-317.

[15] Whitт, W. (2001). The reflection map with discontinuities. Math. Operat. Res. 26, 447-484.

[16] Whit, W. (2002). Stochastic-Process Limits. Springer, New York. 
[17] Williams, R. J. (1995). Semimartingale reflecting Brownian motions in the orthant. In Stochastic Networks (IMA Vol. Math. Appl. 71). Springer, New York, pp. 125-137.

[18] Williams, R. J. (1998). Reflecting diffusions and queueing networks. In Proc. Internat. Congress of Mathematicians, Vol. III (Berlin, 1998), Extra Vol. III, pp. 321-330 (electronic). 